\title{
Improving growth and yield of cowpea by foliar application of chitosan under water stress
}

\author{
S. Farouk ${ }^{1^{*}}$ \& A. Ramadan Amany ${ }^{2}$ \\ 1 Department of Agricultural Botany, Faculty of Agriculture, Mansoura University, Mansoura, Egypt \\ 2 Botany Department, National Research Centre, Dokki, Giza, Egypt
}

\begin{abstract}
Water stress impaired cowpea plant growth and decreased ion percentage and chlorophyll and carbohydrate concentration in the shoot as well as yield and its quality. Foliar-applied chitosan, in particular $250 \mathrm{mg} / \mathrm{l}$, increased plant growth, yield and its quality as well as physiological constituents in plant shoot under stressed or nonstressed conditions as compared to untreated plants. Anatomically, water stress decreased thickness of leaf blade at midrib region, thickness of mesophyll tissue, thickness of midrib vascular bundle. Treatment with chitosan, in particular, 250 $\mathrm{mg} / \mathrm{l}$ and their interactions with stress conditions increased all the above mentioned parameters in either non-stressed or stressed plants. It is suggested that the severity of cowpea plants damaged from water stress was reduced by $250 \mathrm{mg} / \mathrm{l}$ chitosan application.
\end{abstract}

Keywords: water stress, chitosan, cowpea, anatomy, growth, yield.

\section{Introduction}

The world population is increasing at an alarming rate and is expected to reach about six billion by the end of 2050, but food productivity is decreasing due to the effect of various abiotic stresses and climatic change; minimizing these losses is a major area of concern for all nations to cope with increasing food requirements. In the face of a global scarcity of water resources, drought has already become a primary factor in limiting crop production in the world. At present, around $18 \%$ of the global farmland is irrigated (more than 240 million hectares), producing about $40 \%$ of the global food supply (Somerville \& Briscoe 2001). Permanent or temporary water-deficit stress limits the growth and distribution of natural and artificial vegetation and the performance of cultivated plants more than any other environmental factor (Shao et al. 2009). In Egypt, water availability is considered the prime constraint that determines the addition of new cultivated areas. Agricultural expansion needs a huge amount of irrigation water which is already not sufficient to meet all the expected demands.

The responses of plants to drought vary greatly depending on species and stress severity (Mullet \& Whitsitt 1996). Higher plants respond to water deficit in several ways: stomatal closure, leaf rolling, osmotic adjustments, reductions and consequently decreases in cellular expansion, and alterations of various essential physiological and biochemical processes that can affect growth, productivity and yield quality (Costa et al. 2008, Lobato et al. 2008, Hefny 2011). For example, Carvalho et al. (2004) found that lupine cultivars tended to accumulate crude protein and carbon compounds in the seeds at the end of the water stress period (15 days after anthesis), but Jansen (2008) recorded insignificant effect of water stress on protein content when imposed at the same stage.

The sustainable management of water resources is a priority for agriculture also for the temperate regions, e.g. the Mediterranean basin, where dry and hot summers usually occur, and drought events can have a large impact on both productivity and crop quality. Bittelli et al. (2001) reported that occasional or episodic drought events can be counteracted through the use of anti-transpirants, compounds applied to foliage to limit the water loss. They include both film-forming and stomata-closing compounds, able to increase leaf resistance to water vapor

\footnotetext{
* Author for correspondence: tel +0020101105095 email : gadalla@mans.edu.eg
} 
loss, thus improving plant water use in assimilating carbon, and, in turn, producing biomass or yield (Tambussi \& Bort 2007). Another approach to reducing water loss due to transpiration is by increasing the reflection of sunlight from leaves, through reflectant types of antitranspirants, thus limiting water loss from evaporative leaf cooling (Gaballah \& Moursy 2004).

Among anti-transpirant compounds, chitosan has previously proved to be effective in pepper (Bittelli et al. 2001). Chitosan is a natural, low toxic and inexpensive compound that is biodegradable and environmentally friendly with various applications in agriculture; it is obtained by the deacetylation of chitin. In agriculture, chitosan has been used in seed, leaf, fruit and vegetable coatings, as fertilizer and in controlled agrochemical release, to increase plant productivity (New et al. 2004), to protect plants against microorganisms (Farouk et al. 2008) and against oxidative stress (Guan et al. 2009) and to stimulate plant growth (Farouk et al. $2008,2011)$. In the latter studies, a positive effect of chitosan was observed on the growth of roots, shoots and leaves of various plant species. Similar results were determined within sweet pepper and radish (Ghoname et al. 2010, Farouk et al. 2011). In addition, foliar applications with chitosan resulted in higher vegetative growth and improvement in fruit quality of cucumber (Farouk et al. 2008). For other cultivated plants, Bittelli et al. (2001) reported that foliar application of chitosan decreased transpiration in pepper plants, and reduced water use by $26-43 \%$ while maintaining biomass production and yield. Abdel-Mawgoud et al. (2010) on strawberry showed that chitosan application improved plant height, number of leaves, fresh and dry weights of the leaves and yield components. Fruit quality in terms of average weight of individual fruits and total sugars showed similar trends. Recently, Sheikha \& AL-Malki (2011) indicate that application of different concentrations of chitosan enhanced bean shoot and root length, fresh and dry weights of shoots, root and leaf area as well as the level of chlorophyll in leaves. The mechanisms of chitosan in counteracting the harmful effect of water stress are not well understood and there are a few reports in the literature. Transcriptional activation, induced by both chitosan and jasmonic acid, of genes encoding phenylalanine ammonia lyase and protease inhibitors, suggests that chitosan may influence pathways involving jasmonic acid (Doares et al. 1995). Jasmonates exhibit activities similar to the plant hormone abscisic acid, which plays a key role in the regulation of water use by plants. Increased levels of abscisic acid result in closure of stomata and reduced transpiration (Leung \& Giraudat 1998). These authors demonstrated that chitosan inhibited light-induced opening of stomata in tomato and Commelina communis via inducing $\mathrm{H}_{2} \mathrm{O}_{2}$ production in the guard cells. The reported effects of chitosan on stomatal aperture suggest the possibility that it might be a valuable anti-transpirant with useful agricultural applications.

Cowpea (Vigna unguiculata (L) Walp) is one of the ancient grain legumes valued for its nutritional value, especially its high protein content (25\%), flavour and short cooking time (Ogbonnaya et al. 2003). The crop also has ability to maintain soil fertility through its excellent capacity to fix atmospheric nitrogen, and thus does not require very fertile land for growth (Lobato et al. 2006). Cowpea forms an integral part of sustainable agriculture and land use (Ogbonnaya et al. 2003). The total cultivated area of this crop in Egypt was estimated at about 9155 feddan ( $3845 \mathrm{ha}$ ) for dry seed production in 2008, with a mean production of 980 $\mathrm{kg} /$ feddan $\left(412 \mathrm{~kg} \mathrm{ha}^{-1}\right)$. The estimates for fresh pods was $10064 \mathrm{~kg} /$ feddan $\left(4227 \mathrm{~kg} \mathrm{ha}^{-1}\right)$ with a mean production of 5.19 ton/feddan (data from the Department of Agricultural Statistics, Ministry of Agriculture, Giza, Egypt).

Improvements in water economy probably will help water-stressed plants in maintaining their physiological and biochemical processes, at least to an acceptable base line. To the best of our knowledge there has also been no previous report regarding the effects of foliar applied chitosan on cowpea plant growth and yield. Therefore, the objective of this work was to explore its possible role in improving drought tolerance in cowpea plants. 


\section{Materials \& Methods}

Two pot experiments were conducted in the experimental farm and laboratory of the Agricultural Botany Department, Faculty of Agriculture, Mansoura University, Egypt, during the two successive seasons of 2007 and 2008. Cowpea (Vigna ungiculata (L.) Walp. Cv Cream 7) seed was obtained from the Legume Research Institute, Agricultural Research Centre, Ministry of Agriculture, Giza, Egypt. The seeds were sterilized with $1.5 \%$ chlorox, washed three times with distilled water, and then coated with N-fixer okadeen (rhizobia) obtained from the General Organization for Agriculture Equalization Fund, Ministry of Agriculture. Egypt.

Sowing took place on 15 and 10 April in both seasons respectively. Plastic pots $(50 \mathrm{~cm}$ inner diameter and $30 \mathrm{~cm}$ in length) filled with $25 \mathrm{~kg}$ air-dried soil were used. The soil characteristics were as follows: sandy loam in texture, sand, $80 \%$; silt, $15.5 \%$; clay, $4.5 \%$; $\mathrm{pH}$, 7.8; EC, $0.4 \mathrm{dSm}^{-1}$ and organic matter $0.45 \%$. After sowing, irrigation was applied to supply seedlings with $100 \%$ available water, at two-day intervals until the seedlings reached the fourth leaf stage. The seedlings were then thinned to leave seven plants per pot. Phosphorous and potassium fertilizers were added to the soil before sowing at the rate of $5 \mathrm{~g} \mathrm{P}_{2} \mathrm{O}_{5}$ in the form of calcium super phosphate $\left(15.5 \% \mathrm{P}_{2} \mathrm{O}_{5}\right)$ and $2 \mathrm{~g} \mathrm{~K}_{2} \mathrm{O}$ in the form of potassium sulphate (48\%). Ammonium nitrate (33.5\%) was added at the rate of $4 \mathrm{~g} \mathrm{~N} /$ pot in two equal portions; the first during the seedling stage and the second at the start of flowering. After that the pots were divided into three groups for water-stress treatments, with each group divided into four subgroups for chitosan foliar application. The soil moisture for all pots was kept at $80 \%$ field capacity until 15 days after sowing. After that, the water stress treatments were initiated.

Pots were subjected to one of the three water-stress treatments: a well-watered control, $70 \%$ of field capacity; and two water-stress treatments, 'moderate' at 50\% and 'severe' at 30\% of field capacity. In the stressed treatments, moisture levels were allowed to fall from the initial $70 \%$ to $50 \%$ and $30 \%$ of field capacity, respectively. All pots were weighed every two days. Losses in pot weight represent transpiration and evaporation. Cumulative water losses were added to each pot to compensate for transpiration and evaporation. Accumulated water loss was calculated as the differences in pot weight between successive weightings. At 40, 50 and 60 days from sowing, the plants were sprayed with either tap water or chitosan at 125, 250 or $500 \mathrm{mg} / \mathrm{l}$ until dripping, using a small pressure pump after adding tween 20 as a wetting agent at a concentration of $0.5 \%$.

Three uniform plants were uprooted from each pot at the full blooming stage ( 80 days from sowing) to measure morphological and physiological characteristics as well as leaflet anatomy. For morphological measures, the plants were cleaned and plant height, number of leaves, number of branches and leaf area (using a leaf-area meter) were determined. Fresh and dry weights were estimated by drying each plant at $70^{\circ} \mathrm{C}$ to a constant weight. Chemical composition measures included total chlorophyll, carbohydrates and minerals. Total chlorophyll was extracted for $24 \mathrm{~h}$ at room temperature in methanol after adding traces of sodium carbonate, and determined spectrophotometrically (Spekol 11, Uk) according to Lichtenthaler \& Wellburn (1983). Total carbohydrate content was estimated using the anthrone method as described by Sadasivam \& Manickam (1996). To measure mineral constituents, a dry shoot $(0.2 \mathrm{~g})$ was digested using $5 \mathrm{ml}$ of a mixture of sulfuric and perchloric acid $\left(\mathrm{HClO}_{3} / \mathrm{H}_{2} \mathrm{SO}_{4}\right.$ 1:1 v:v) until the sample became clear and cool; this was made up to $50 \mathrm{ml}$ using deionized water. Total nitrogen was determined by the micro-Kjeldahl method. Potassium was determined by flame-photometry (Kalra 1998). Phosphorous was estimated using ammonium molybdate and ascorbic acid (Cooper 1977).

For leaflet anatomy, small pieces from the midrib region of the $3^{\text {rd }}$ upper leaflet (second season) were fixed in formalin aceto alcohol for $48 \mathrm{~h}$, then dehydrated in an n-butanole series and embedded in paraffin wax $\left(52-54{ }^{\circ} \mathrm{C}\right.$ melting points). Sections were prepared using a 
rotary microtome at 15-17 $\mu \mathrm{m}$ thickness and stained with safranin/light green and finally mounted in Canada balsam. Selected sections were examined under a microscope to determine any anatomical changes in leaf structure.

At harvest time (140 days from sowing) the total yield per plant was recorded. Seed quality was represented by the concentrations of nitrogen, phosphorous, potassium, protein and carbohydrates determined in the dry seeds as previously described in shoots. Finally, the protein percentage in dry seeds was measured by multiplying nitrogen content by 6.25 .

All data were analyzed statistically using one-way ANOVA, followed by Duncan's Multiple Range Test using COSTAT software. The values presented are all mean \pm SD for three samples in each group. $\mathrm{P}$ values $<0.05$ were considered as significant.

\section{Results}

Generally, severe reductions in plant growth, manifested by smaller, chlorotic, wilted, and rolled leaves were recorded due to water stress (Table 1). All plant growth characters (plant height, shoot and leaf number per plant, and shoot fresh and dry weight) significantly decreased due to water stress in both growing seasons. The largest reduction was observed under severe water stress ( $30 \%$ of field capacity).

Table 1: Effect of water stress, chitosan and their interaction on growth parameters during two growing seasons $\left(1^{\text {st }}\right.$ and $\left.2^{\text {nd }}\right)$. Water stress values are $\%$ of field capacity; chitosan treatments are in $\mathrm{mg} \mathrm{l}^{-1}$

\begin{tabular}{|c|c|c|c|c|c|c|c|c|c|c|c|}
\hline \multicolumn{2}{|c|}{ Treatments } & \multicolumn{2}{|c|}{ Plant ht $(\mathrm{cm})$} & \multicolumn{2}{|c|}{ Branch number } & \multicolumn{2}{|c|}{ Leaf number } & \multicolumn{2}{|c|}{ Shoot dry wt (g) } & \multicolumn{2}{|c|}{ Shoot fresh wt (g) } \\
\hline $\begin{array}{l}\text { Water } \\
\text { stress }\end{array}$ & Chitosan & $1^{\text {st }}$ & $2^{\text {nd }}$ & $1^{\mathrm{st}}$ & $2^{\text {nd }}$ & $1^{\mathrm{st}}$ & $2^{\text {nd }}$ & $1^{\mathrm{st}}$ & $2^{\text {nd }}$ & $1^{\mathrm{st}}$ & $2^{\text {nd }}$ \\
\hline \multirow[t]{4}{*}{70} & water & $38.1 \pm 0.3^{\mathrm{d}}$ & $37.5 \pm 2.1^{\mathrm{d}}$ & $40+00^{\circ}$ & $4.0 \pm 0.0^{\mathrm{c}}$ & $30.0 \pm 1.1^{\mathrm{e}}$ & $29.0 \pm 0^{\mathrm{de}}$ & $7.2 \pm 0.1^{\mathrm{d}}$ & $7.1 \pm 0.5^{c}$ & $37.2 \pm 0.4^{\mathrm{def}}$ & $37.5 \pm 0.5^{\mathrm{de}}$ \\
\hline & 125 & $.1 \pm 1.5^{\mathrm{b}}$ & $41.1 \pm 1.2^{\mathrm{b}}$ & $5.0 \pm 0.0^{\mathrm{b}}$ & $4.7 \pm 1.2^{\mathrm{b}}$ & $34.3 \pm 1.2^{\mathrm{c}}$ & $34.3 \pm 1.2^{\mathrm{b}}$ & $7.8 \pm 0.1^{\mathrm{c}}$ & $7.7 \pm 0.1^{\mathrm{b}}$ & $40.3 \pm 0.8^{\mathrm{c}}$ & $40.0 \pm 1.8^{\mathrm{c}}$ \\
\hline & 250 & $43.8 \pm 2.0^{\mathrm{a}}$ & $43.3 \pm 1.9^{\mathrm{a}}$ & $6.0 \pm 0.0^{\mathrm{a}}$ & $5.7 \pm 1.2^{\mathrm{a}}$ & $37.7 \pm 1.2^{\mathrm{a}}$ & $37.3 \pm 1.2^{\mathrm{a}}$ & $8.7 \pm 0.7^{\mathrm{a}}$ & $8.5 \pm 1.1^{\mathrm{a}}$ & $56.9 \pm 4.4^{\mathrm{a}}$ & $47.0 \pm 4.3^{\mathrm{a}}$ \\
\hline & 500 & $36.0 \pm 2.4^{\mathrm{e}}$ & $35.5 \pm 2.5^{\mathrm{e}}$ & $4.0 \pm 0.0^{\mathrm{c}}$ & $4.0 \pm 0.0^{\mathrm{c}}$ & $28.3 \pm 1.2^{\mathrm{f}}$ & $27.7 \pm 1.2^{\mathrm{e}}$ & $6.9 \pm 0.2^{\mathrm{e}}$ & $6.7 \pm 0.1^{\mathrm{d}}$ & $37.0 \pm 1.0^{\mathrm{def}}$ & $36.7 \pm 1.2^{\mathrm{def}}$ \\
\hline \multirow[t]{4}{*}{50} & water & $30.3 \pm 2.7^{\mathrm{g}}$ & $29.8 \pm 1.1^{\mathrm{g}}$ & $3.0 \pm 0.0^{\mathrm{d}}$ & $3.0 \pm 0.0^{\mathrm{d}}$ & $25.3 \pm 1.2^{\mathrm{h}}$ & $24.7 \pm 1.2^{\mathrm{fg}}$ & $6.6 \pm 0.2^{\mathrm{f}}$ & $6.1 \pm 0.02^{\mathrm{e}}$ & $35.8 \pm 1.6^{\mathrm{fg}}$ & $35.6 \pm 1.0^{\text {ef }}$ \\
\hline & 125 & $39.0 \pm 1.6^{\mathrm{cd}}$ & $39.0 \pm 0.5^{\mathrm{c}}$ & $4.0 \pm 0.0^{\mathrm{c}}$ & $4.0 \pm 0.0^{\mathrm{c}}$ & $30.7 \pm 1.2^{\mathrm{e}}$ & $30.0 \pm 0.0^{\mathrm{cd}}$ & $7.3 \pm 0.1^{\mathrm{d}}$ & $7.3 \pm 0.1^{\mathrm{c}}$ & $38.1 \pm 1.6^{\mathrm{de}}$ & $37.9 \pm 0.8^{\text {cde }}$ \\
\hline & 250 & $42.5 \pm 2.6^{\mathrm{a}}$ & $42.0 \pm 1.1^{\mathrm{b}}$ & $5.0 \pm 0.0^{\mathrm{b}}$ & $5.0 \pm 0.0^{\mathrm{b}}$ & $35.7 \pm 1.2^{\mathrm{b}}$ & $35.7 \pm 1.2^{\mathrm{b}}$ & $8.2 \pm 0.3^{\mathrm{b}}$ & $7.8 \pm 0.1^{\mathrm{b}}$ & $44.1 \pm 2.1^{\mathrm{b}}$ & $43.3 \pm 3.2^{\mathrm{b}}$ \\
\hline & 500 & $32.4 \pm 3.2^{\mathrm{f}}$ & $31.8 \pm 0.9^{\mathrm{f}}$ & $3.7 \pm 1.2^{\mathrm{c}}$ & $3.0 \pm 0.0^{\mathrm{d}}$ & $26.7 \pm 1.2^{\mathrm{g}}$ & $26.0 \pm 2.0^{\mathrm{f}}$ & $6.7 \pm 0.05^{\mathrm{f}}$ & $6.7 \pm 0.02^{\mathrm{d}}$ & $36.6 \pm 0.7^{\mathrm{ef}}$ & $36.6 \pm 1.0^{\mathrm{def}}$ \\
\hline \multirow[t]{4}{*}{30} & water & $26.3 \pm 1.6^{\mathrm{i}}$ & $25.3 \pm 2.2^{\mathrm{i}}$ & $2.0 \pm 0.0^{\mathrm{e}}$ & $2.0 \pm 0.0^{\mathrm{e}}$ & $21.0 \pm 2.0^{\mathrm{j}}$ & $21.3 \pm 4.6^{\mathrm{h}}$ & $5.2 \pm 0.1^{\mathrm{i}}$ & $5.0 \pm 0.02^{\mathrm{g}}$ & $29.4 \pm 2.5^{\mathrm{i}}$ & $28.3 \pm 5.3^{\mathrm{h}}$ \\
\hline & 125 & $28.9 \pm 0.2^{\mathrm{gh}}$ & $28.1 \pm 0.2^{\mathrm{h}}$ & $3.0 \pm 0.0^{\mathrm{d}}$ & $3.0 \pm 0.0^{\mathrm{d}}$ & $24.3 \pm 1.2^{\mathrm{h}}$ & $23.3 \pm 1.2^{\mathrm{g}}$ & $6.0 \pm 0.1^{\mathrm{g}}$ & $5.8 \pm 0.2^{\mathrm{f}}$ & $34.4 \pm 1.0^{\mathrm{g}}$ & $34.4 \pm 2.6^{\mathrm{f}}$ \\
\hline & 250 & & $39.6 \pm 1.5^{\mathrm{c}}$ & $4.0 \pm 0.0^{\mathrm{c}}$ & $4.0 \pm 0.0^{\mathrm{c}}$ & $32.0 \pm 0.0^{\mathrm{d}}$ & $31.3 \pm 1.2^{\mathrm{c}}$ & $7.4 \pm 0.1^{\mathrm{d}}$ & $7.4 \pm 0.1^{\mathrm{c}}$ & $38.5 \pm 0.7^{\mathrm{d}}$ & $38.2 \pm 0.5^{\mathrm{cd}}$ \\
\hline & 500 & $27.5 \pm 1.3^{\mathrm{hi}}$ & $27.5 \pm 0.4^{\mathrm{h}}$ & $2.7 \pm 1.2^{\mathrm{d}}$ & $2.3 \pm 1.2^{\mathrm{e}}$ & $22.3 \pm 1.2^{\mathrm{i}}$ & $21.7 \pm 1.2^{\mathrm{h}}$ & $5.4 \pm 0.1^{\mathrm{h}}$ & $5.3 \pm 0.1^{\mathrm{g}}$ & $32.4 \pm 3.2^{\mathrm{h}}$ & $31.5 \pm 2.7^{\mathrm{g}}$ \\
\hline
\end{tabular}

Values are given as mean \pm SD of three replicates. Means in columns with different letters are

significantly different at $\mathrm{P}<0.05$ by Duncan's Multiple Range Test.

Foliar application of chitosan, and especially at $250 \mathrm{mg} \mathrm{l}^{-1}$, improved all plant growth measures compared to untreated control plants. Regarding the interaction effects, application of chitosan at $250 \mathrm{mg} \mathrm{l}^{-1}$ significantly increased all growth parameter of cowpea with an increasing effect with greater stress. However, while application at $125 \mathrm{mg} \mathrm{l}^{-1}$ also counteracted the harmful effect of water stress on plant growth, application at $500 \mathrm{mg} \mathrm{l}^{-1}$ significantly decreased all the parameters.

The total content of chlorophylls and carbohydrates (Table 2) significantly decreased under water stress in both growing seasons as compared with the control plants. Foliar application of chitosan, especially at $250 \mathrm{mg} \mathrm{l}^{-1}$, significantly increased these parameters compared with untreated plants under stress. As for its interactions with water stress, the effect of chitosan in counteracting the water stress decreased with increasing water stress. 
Table 2: Effect of water stress and chitosan and their interactions on chlorophyll (mg/g fresh wt) and total carbohydrates $(\mathrm{mg} / \mathrm{g}$ dry $\mathrm{wt})$ contents during the two growing seasons $\left(1^{\text {st }}\right.$ and $\left.2^{\text {nd }}\right)$. Water stress values are $\%$ of field capacity; chitosan treatments are in $\mathrm{mg}^{-1}$

\begin{tabular}{|c|c|c|c|c|c|c|c|c|c|}
\hline \multicolumn{2}{|c|}{ Treatments } & \multicolumn{2}{|c|}{ Chlorophyll A } & \multicolumn{2}{|c|}{ Chlorophyll B } & \multicolumn{2}{|c|}{ Total chlorophyll } & \multicolumn{2}{|c|}{ Total carbohydrates } \\
\hline $\begin{array}{l}\text { Water } \\
\text { stress }\end{array}$ & Chitosan & $1^{\mathrm{st}}$ & $2^{\text {nd }}$ & $1^{\mathrm{st}}$ & $2^{\text {nd }}$ & $1^{\text {st }}$ & $2^{\text {nd }}$ & $1^{\mathrm{st}}$ & $2^{\text {nd }}$ \\
\hline \multirow[t]{4}{*}{70} & water & $043+0,3^{b c}$ & $0.51 \pm 0.22^{\mathrm{bc}}$ & $0.30 \pm 0.01^{\mathrm{a}}$ & $0.19 \pm 0.0$ & $0.73 \pm 0.03^{\text {de }}$ & $0.70 \pm 0$ & $30.2 \pm 0.6^{\mathrm{e}}$ & $32.0 \pm 1$. \\
\hline & 12 & & & & $0.29 \pm$ & $0.81 \pm 0.02^{\mathrm{bc}}$ & & $35.0 \pm 1.8^{\mathrm{bc}}$ & \\
\hline & & & $9^{\mathrm{a}}$ & $08^{\mathrm{a}}$ & $0.28 \pm$ & $1.04 \pm 0.30^{\mathrm{a}}$ & $1.00 \pm 0.20^{\mathrm{a}}$ & $7.3 \pm 0.9^{\mathrm{a}}$ & \\
\hline & 500 & & & $26^{\mathrm{a}}$ & 0 . & 0.70 & $0.68 \pm 0.02^{\mathrm{de}}$ & $27.2 \pm 4.6^{\mathrm{f}}$ & $1.4^{\mathrm{cd}}$ \\
\hline \multirow[t]{4}{*}{50} & & & & $0.25 \pm 0$ & 0.2 & $0.64 \pm 0 .($ & $0.60 \pm$ & $25.5 \pm 0.4^{\mathrm{f}}$ & \\
\hline & & & & $0.25 \pm$ & & & & & \\
\hline & 25 & & 0.72 & & $5^{\mathrm{a}}$ & & $0.87 \pm 0.07^{\mathrm{b}}$ & $36.3 \pm 0.9^{\mathrm{ab}}$ & $37.5 \pm 1.1^{\mathrm{a}}$ \\
\hline & 500 & 0.5 & & $0.14 \pm 0.28^{\mathrm{a}}$ & $0.23 \pm 0$ & $0.67 \pm 0.03^{\mathrm{efg}}$ & $0.63 \pm 0.05^{\mathrm{ef}}$ & $27.3 \pm 0.9^{f}$ & $1.3^{\mathrm{de}}$ \\
\hline \multirow[t]{4}{*}{30} & & & & & & & & & \\
\hline & & & & & & & & & \\
\hline & 25 & & $0.51 \pm$ & $0.22 \pm 0$ & $0.24 \pm 0$ & $0.77 \pm 0$ & $0.75 \pm 0.04^{\mathrm{cd}}$ & $33.3 \pm 0.5^{\mathrm{cd}}$ & $35.5 \pm 0.4^{\mathrm{ab}}$ \\
\hline & 500 & $0.43 \pm 0.27^{\mathrm{bc}}$ & $0.32 \pm 0.25^{\mathrm{cd}}$ & $0.11 \pm 0.28^{\mathrm{a}}$ & $0.20 \pm 0.31^{\mathrm{a}}$ & $0.54 \pm 0.05^{\mathrm{h}}$ & $0.52 \pm 0.06^{\mathrm{g}}$ & $21.8 \pm 1.8^{\mathrm{g}}$ & $24.3 \pm 1.6^{\mathrm{f}}$ \\
\hline
\end{tabular}

Values are given as mean \pm SD of three replicates. Means in columns with different letters are significantly different at $\mathrm{P}<0.05$ by Duncan's Multiple Range Test.

Water stress is generally recognized as injurious to plants by disturbing the electrolyte balance, resulting in deficiency of some nutrients. Water stress decreased significantly the percentages of nitrogen, phosphorus and potassium in both growing seasons (Table 3). The largest reduction occurred under severe water stress. Pronounced and highly significant increases in all these nutrients occurred after exogenous application of chitosan, especially at $250 \mathrm{mg}^{-1}$. As with other measures, application at $500 \mathrm{mg} \mathrm{l}^{-1}$ reduced this effect. The impact of chitson was greater in stressed than unstressed plants.

Table 3: Effect of water stress and chitosan and their interactions on nitrogen,phosphorous and potassium percentage during the two growing seasons $\left(1^{\text {st }}\right.$ and $\left.2^{\text {nd }}\right)$. Water stress values are $\%$ of field capacity; chitosan treatments are in $\mathrm{mg} \mathrm{l}^{-1}$

\begin{tabular}{|c|c|c|c|c|c|c|c|}
\hline \multicolumn{2}{|l|}{ Treatments } & \multicolumn{2}{|l|}{ Nitrogen $\%$} & \multicolumn{2}{|c|}{ Phosphorous \% } & \multicolumn{2}{|l|}{ Potassium\% } \\
\hline Water stress & Chitosan & $1^{\text {st }}$ & $2^{\text {nd }}$ & $1^{\text {st }}$ & $2^{\text {nd }}$ & $1^{\mathrm{st}}$ & $2^{\text {nd }}$ \\
\hline \multirow[t]{4}{*}{70} & water & $3.71 \pm 0.14^{f}$ & $3.43 \pm 0.14^{\mathrm{e}}$ & $0.50 \pm 0.01^{\mathrm{cd}}$ & $0.51 \pm 0.01^{\mathrm{e}}$ & $1.25 \pm 0.07^{\mathrm{e}}$ & $1.04 \pm 0.08^{\mathrm{e}}$ \\
\hline & 125 & $4.29 \pm 0.08^{c}$ & $4.17 \pm 0.08^{b}$ & $0.54 \pm 0.03^{b c}$ & $0.61 \pm 0.04^{\mathrm{c}}$ & $1.56 \pm 0.03^{b}$ & $1.44 \pm 0.10^{\mathrm{b}}$ \\
\hline & 250 & $4.76 \pm 0.14^{\mathrm{a}}$ & $4.48 \pm 0.14^{\mathrm{a}}$ & $0.72 \pm 0.16^{\mathrm{a}}$ & $0.76 \pm 0.03^{\mathrm{a}}$ & $1.68 \pm 0.02^{\mathrm{a}}$ & $1.59 \pm 0.05^{\mathrm{a}}$ \\
\hline & 500 & $3.38 \pm 0.16^{\mathrm{g}}$ & $3.22 \pm 0.14^{\mathrm{f}}$ & $0.48 \pm 0.02^{\mathrm{de}}$ & $0.49 \pm 0.02^{\mathrm{ef}}$ & $1.10 \pm 0.11^{\mathrm{f}}$ & $0.91 \pm 0.05^{\mathrm{f}}$ \\
\hline \multirow[t]{4}{*}{50} & water & $3.12 \pm 0.08^{h}$ & $3.01 \pm 0.14^{\mathrm{gh}}$ & $0.43 \pm 0.02^{\mathrm{efg}}$ & $0.45 \pm 0.02^{\mathrm{g}}$ & $0.85 \pm 0.07^{\mathrm{h}}$ & $0.78 \pm 0.03^{h}$ \\
\hline & 125 & $3.99 \pm 0.14^{\mathrm{e}}$ & $3.64 \pm 0.24^{\mathrm{d}}$ & $0.51 \pm 0.01^{\mathrm{cd}}$ & $0.52 \pm 0.02^{\mathrm{e}}$ & $1.37 \pm 0.10^{\mathrm{d}}$ & $1.19 \pm 0.09^{\mathrm{d}}$ \\
\hline & 250 & $4.50 \pm 0.21^{\mathrm{b}}$ & $4.29 \pm 0.08^{b}$ & $0.59 \pm 0.04^{\mathrm{b}}$ & $0.70 \pm 0.05^{\mathrm{b}}$ & $1.64 \pm 0.05^{\mathrm{a}}$ & $1.54 \pm 0.05^{\mathrm{a}}$ \\
\hline & 500 & $3.19 \pm 0.08^{h}$ & $3.10 \pm 0.08^{\mathrm{fg}}$ & $0.46 \pm 0.03^{\mathrm{def}}$ & $0.47 \pm 0.01^{\mathrm{fg}}$ & $0.97 \pm 0.07^{\mathrm{g}}$ & $0.84 \pm 0.05^{\mathrm{g}}$ \\
\hline \multirow[t]{4}{*}{30} & water & $2.68 \pm 0.08^{\mathrm{k}}$ & $2.49 \pm 0.21^{\mathrm{i}}$ & $0.37 \pm 0.05^{\mathrm{g}}$ & $0.38 \pm 0.03^{\mathrm{i}}$ & $0.70 \pm 0.08^{\mathrm{j}}$ & $0.63 \pm 0.06^{\mathrm{j}}$ \\
\hline & 125 & $3.01 \pm 0.14^{\mathrm{i}}$ & $2.87 \pm 0.14^{\mathrm{h}}$ & $0.40 \pm 0.02^{\mathrm{fg}}$ & $0.42 \pm 0.02^{\mathrm{h}}$ & $0.81 \pm 0.05^{\mathrm{hi}}$ & $0.73 \pm 0.03^{h i}$ \\
\hline & 250 & $4.17 \pm 0.08^{\mathrm{d}}$ & $3.92 \pm 0.28^{c}$ & $0.50 \pm 0.13^{\mathrm{cd}}$ & $0.55 \pm 0.04^{\mathrm{d}}$ & $1.49 \pm 0.10^{\mathrm{c}}$ & $1.30 \pm 0.07^{\mathrm{c}}$ \\
\hline & 500 & $2.84 \pm 0.21^{\mathrm{j}}$ & $2.63 \pm 0.22^{\mathrm{i}}$ & $0.39 \pm 0.01^{\mathrm{g}}$ & $0.40 \pm 0.01^{\mathrm{hi}}$ & $0.75 \pm 0.02^{\mathrm{ij}}$ & $0.70 \pm 0.02^{\mathrm{i}}$ \\
\hline
\end{tabular}

Values are given as mean \pm SD of three replicates. Means in columns with different letters are significantly different at $\mathrm{P}<0.05$ by Duncan's Multiple Range Test.

Cross-sections of the $3^{\text {rd }}$ terminal leaflet of cowpea showed that there were significant changes in leaf anatomical characteristics due to water stress (Table 4, Fig. 1). In particular, water stress resulted in a significant decrease of the thickness of almost all anatomical characters, i.e. the thickness of the leaflet, mesophyll tissue as well as main vascular bundle dimensions, xylem and phloem tissue thickness. 
Table 4: Effect of water stress and chitosan and their interaction on leaflet anatomical characters of cowpea plants in the second season. Water stress values are $\%$ of field capacity; chitosan treatments are in $\mathrm{mg}^{-1}$

\begin{tabular}{|c|c|c|c|c|c|c|c|c|c|c|}
\hline \multicolumn{2}{|c|}{ Treatments } & \multirow{2}{*}{ 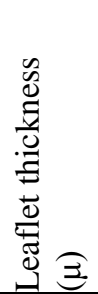 } & \multirow{2}{*}{ 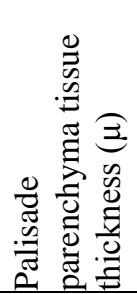 } & \multirow{2}{*}{ 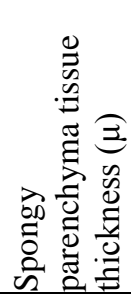 } & \multirow{2}{*}{ 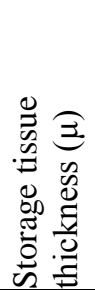 } & \multirow{2}{*}{ 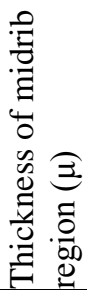 } & \multicolumn{2}{|c|}{$\begin{array}{l}\text { Main vascular } \\
\text { bundle } \\
\text { dimension }(\mu)\end{array}$} & \multirow{2}{*}{ 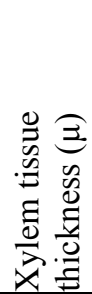 } & \multirow{2}{*}{ 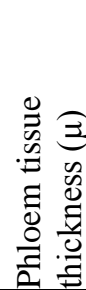 } \\
\hline $\begin{array}{l}\text { Water } \\
\text { stress }\end{array}$ & Chitosan & & & & & & length & width & & \\
\hline \multirow[t]{4}{*}{70} & water & 68 & 32 & 36 & 16 & 276 & 48 & 96 & 36 & 12 \\
\hline & 125 & 68 & 36 & 32 & 24 & 328 & 60 & 104 & 44 & 16 \\
\hline & 250 & 68 & 36 & 32 & 44 & 336 & 60 & 116 & 40 & 20 \\
\hline & 500 & 56 & 32 & 24 & 32 & 284 & 52 & 112 & 32 & 20 \\
\hline \multirow[t]{4}{*}{50} & water & 52 & 28 & 24 & 24 & 216 & 32 & 68 & 24 & 8 \\
\hline & 125 & 72 & 40 & 32 & 44 & 308 & 52 & 124 & 36 & 16 \\
\hline & 250 & 72 & 36 & 36 & 52 & 316 & 56 & 128 & 40 & 16 \\
\hline & 500 & 56 & 32 & 24 & 40 & 316 & 56 & 92 & 44 & 12 \\
\hline \multirow[t]{4}{*}{30} & water & 48 & 24 & 24 & 16 & 188 & 28 & 60 & 20 & 8 \\
\hline & 125 & 56 & 36 & 20 & 28 & 268 & 36 & 88 & 20 & 16 \\
\hline & 250 & 60 & 28 & 32 & 44 & 324 & 48 & 96 & 32 & 16 \\
\hline & 500 & 52 & 28 & 24 & 20 & 212 & 36 & 68 & 24 & 12 \\
\hline
\end{tabular}
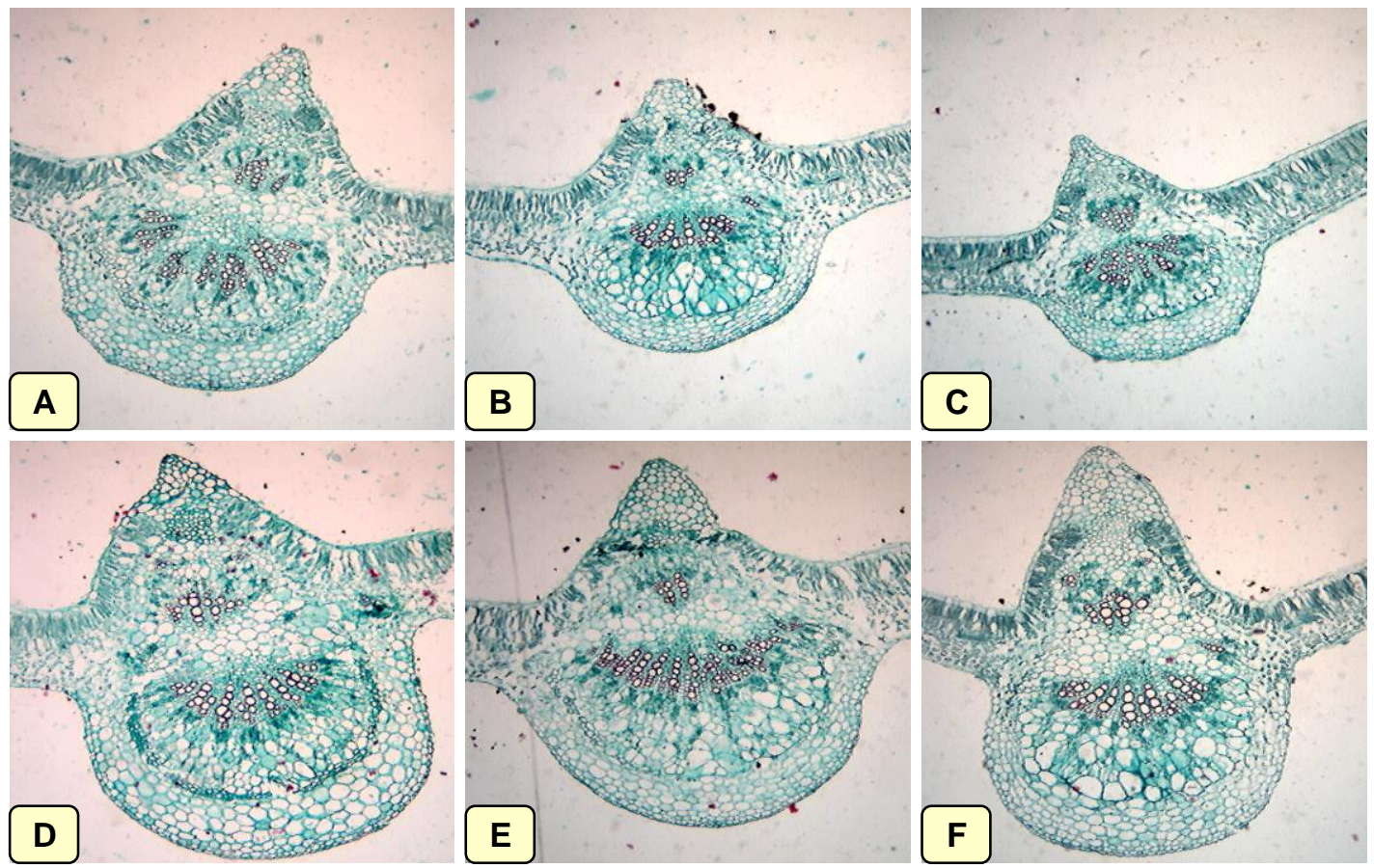

Figure 1: Effect of water stress and chitosan and their interaction on leaflet anatomical characters of cowpea plants in the second season. A, 70\% of field capacity; B, $50 \%$ of field capacity; C, $30 \%$ of field capacity; D, $70 \%$ of field capacity $+250 \mathrm{mg} / \mathrm{l}$ chitosan; E, $50 \%$ of field capacity $+250 \mathrm{mg} / \mathrm{l}$ chitosan; E, $30 \%$ of field capacity $+250 \mathrm{mg} / \mathrm{l}$ chitosan. (all 40x). 
Table 5: Effect of water stress and chitosan and their interactions on yield and its quality during the two growing seasons $\left(1^{\text {st }}\right.$ and $\left.2^{\text {nd }}\right)$. Water stress values are $\%$ of field capacity; chitosan treatments are in $\mathrm{mg}^{-1}$.

\begin{tabular}{|c|c|c|c|c|c|c|c|c|c|c|c|c|c|}
\hline \multicolumn{2}{|c|}{ Treatments } & \multicolumn{2}{|c|}{ Pod yield per plant } & \multicolumn{2}{|l|}{ Nitrogen $\%$} & \multicolumn{2}{|l|}{ Phosphorous \% } & \multicolumn{2}{|l|}{ Potassium \% } & \multicolumn{2}{|l|}{ Protein $\%$} & \multicolumn{2}{|c|}{ Carbohydrates \% } \\
\hline $\begin{array}{l}\text { Water } \\
\text { stress }\end{array}$ & Chitosan & $1^{\mathrm{st}}$ & $2^{\text {nd }}$ & $1^{\mathrm{st}}$ & $2^{\text {nd }}$ & $1^{\mathrm{st}}$ & $2^{\text {nd }}$ & $1^{\mathrm{st}}$ & $2^{\text {nd }}$ & $1^{\mathrm{st}}$ & $2^{\text {nd }}$ & $1^{\mathrm{st}}$ & $2^{\text {nd }}$ \\
\hline \multirow[t]{4}{*}{70} & water & $16.5 \pm 0.7^{\mathrm{bcd}}$ & $16.5 \pm 0.5^{\mathrm{d}}$ & $3.87 \pm 0.70^{\text {bcde }}$ & $3.87 \pm 0.70^{\text {cde }}$ & $0.53 \pm 0.02^{\mathrm{de}}$ & $0.51 \pm 0.02^{\mathrm{de}}$ & $1.26 \pm 0.03^{\mathrm{cde}}$ & $1.16 \pm 0.21^{\mathrm{def}}$ & $24.2 \pm 4.4^{\text {bcde }}$ & $24.2 \pm 4.4^{\text {cde }}$ & $38.8 \pm 0.3^{\mathrm{e}}$ & $38.3 \pm 0.2$ \\
\hline & 125 & $17.7 \pm 0.4^{\mathrm{bc}}$ & $17.4 \pm 0.4^{\mathrm{bc}}$ & $4.13 \pm 1.26^{\mathrm{abc}}$ & $4.10 \pm 0.08^{\mathrm{bc}}$ & $0.61 \pm 0.07^{\mathrm{c}}$ & $0.62 \pm 0.07^{\mathrm{c}}$ & $1.36 \pm 0.14^{\mathrm{bc}}$ & $1.34 \pm 0.20^{\mathrm{bc}}$ & $25.8 \pm 7.9^{\mathrm{abc}}$ & & $41.1 \pm 0.8^{c}$ & $41.6 \pm 0.5^{\mathrm{b}}$ \\
\hline & 250 & $22.6 \pm 5.4^{\mathrm{a}}$ & $19.2 \pm 1.8^{\mathrm{a}}$ & $4.50 \pm 0.21^{\mathrm{a}}$ & $4.45 \pm 0.21^{\mathrm{a}}$ & $0.87 \pm 0.22^{\mathrm{a}}$ & $0.84 \pm 0.12^{\mathrm{a}}$ & $1.55 \pm 0.08^{\mathrm{a}}$ & $1.51 \pm 0.07^{\mathrm{a}}$ & $28.1 \pm 1.3^{\mathrm{a}}$ & $27.8 \pm 1.3^{\mathrm{a}}$ & $44.9 \pm 2.6^{\mathrm{a}}$ & $43.8 \pm 0.7^{a}$ \\
\hline & 500 & $16.6 \pm 0.6^{\text {cde }}$ & $16.6 \pm 0.5^{\mathrm{cd}}$ & $3.75 \pm 0.49^{\text {bcdef }}$ & $3.75 \pm 0.08^{\mathrm{def}}$ & $0.51 \pm 0.01^{\mathrm{def}}$ & $0.51 \pm 0.01^{\mathrm{de}}$ & $1.19 \pm 0.28^{\mathrm{def}}$ & $1.10 \pm 0.02^{\mathrm{efg}}$ & $23.5 \pm 3.1^{\text {bcdef }}$ & $23.5 \pm 0.5^{\mathrm{def}}$ & $37.9 \pm 0.8^{f}$ & $37.3 \pm 0.8^{\mathrm{e}}$ \\
\hline \multirow[t]{4}{*}{50} & water & $15.3 \pm 0.3^{\text {ef }}$ & $15.7 \pm 0.6^{\mathrm{e}}$ & $3.52 \pm 0.08^{\operatorname{defg}}$ & $3.47 \pm 0.08^{\mathrm{fgh}}$ & $0.47 \pm 0.002^{\text {efgh }}$ & $0.47 \pm 0.01^{\mathrm{ef}}$ & $1.09 \pm 0.18^{\mathrm{fgh}}$ & $1.01 \pm 0.15^{\mathrm{ghi}}$ & $22.0 \pm 0.5^{\text {defg }}$ & $21.7 \pm 0.51^{\mathrm{fgh}}$ & $35.3 \pm 0.4^{\mathrm{h}}$ & $34.8 \pm 1.2^{2}$ \\
\hline & 125 & $16.5 \pm 0.5^{\text {cde }}$ & $16.5 \pm 0.6^{\mathrm{d}}$ & $3.92 \pm 0.84^{\text {bcde }}$ & $3.92 \pm 0.84^{\mathrm{cd}}$ & $0.56 \pm 0.03^{\mathrm{cd}}$ & $0.54 \pm 0.01^{\mathrm{d}}$ & $1.31 \pm 0.03^{\mathrm{cd}}$ & $1.22 \pm 0.02^{\text {cde }}$ & $24.4 \pm 5.3^{\text {bcde }}$ & $24.5 \pm$ & $39.7 \pm 0.7^{\mathrm{d}}$ & $38.8 \pm 0.5$ \\
\hline & 250 & $18.2 \pm 0.6^{\mathrm{b}}$ & $17.8 \pm 1.0^{\mathrm{b}}$ & $4.27 \pm 0.14^{\mathrm{ab}}$ & $4.24 \pm 0.08^{\mathrm{ab}}$ & $0.72 \pm 0.13^{\mathrm{b}}$ & $0.69 \pm 0.06^{\mathrm{b}}$ & $1.45 \pm 0.15^{\mathrm{ab}}$ & $1.39 \pm 0.13^{\mathrm{ab}}$ & $26.7 \pm 0.9^{\mathrm{ab}}$ & $26.5 \pm 1.5^{\mathrm{ab}}$ & $42.7 \pm 0.2^{b}$ & $42.2 \pm 0.3^{b}$ \\
\hline & 500 & $15.3 \pm 0.5^{\mathrm{ef}}$ & $15.5 \pm 0.8^{\mathrm{e}}$ & $3.59 \pm 0.16^{\text {cdef }}$ & $3.57 \pm 0.24^{\mathrm{efg}}$ & $0.50 \pm 0.01^{\mathrm{defg}}$ & $0.50 \pm 0.01^{\mathrm{e}}$ & $1.14 \pm 0.23^{\mathrm{efg}}$ & $1.04 \pm 0.10^{\text {fgh }}$ & $22.4 \pm 1.0^{\mathrm{cdef}}$ & $22.3 \pm 1.8^{\mathrm{efg}}$ & $36.5 \pm 0.5^{\mathrm{g}}$ & $35.9 \pm 0.7^{\mathrm{f}}$ \\
\hline \multirow[t]{4}{*}{30} & water & $13.2 \pm 0.9^{\mathrm{g}}$ & $12.9 \pm 0.2^{\mathrm{g}}$ & $2.98 \pm 0$ & $2.98 \pm$ & $0.42 \pm 0.03^{\mathrm{h}}$ & $0.41 \pm$ & $0.92 \pm 0.15^{\mathrm{i}}$ & & $18.7 \pm$ & & $30.0 \pm 0.5^{\mathrm{k}}$ & $28.7 \pm 0.5^{\mathrm{j}}$ \\
\hline & 125 & $15.6 \pm 0.3^{\mathrm{def}}$ & $15.4 \pm 1.7^{\mathrm{e}}$ & $3.38 \pm 1.12^{\mathrm{efg}}$ & $3.38 \pm 0.08^{\mathrm{gh}}$ & $0.45 \pm 0.00^{\mathrm{fgh}}$ & $0.44 \pm 0.03^{\mathrm{fg}}$ & $1.04 \pm 0.10^{\text {ghi }}$ & $0.94 \pm 0.25^{\mathrm{hij}}$ & $21.1 \pm 7.0^{\mathrm{efg}}$ & $21.1 \pm 0.50^{\mathrm{gh}}$ & $33.2 \pm 0.5^{\mathrm{i}}$ & $32.5 \pm 1.2^{\mathrm{h}}$ \\
\hline & 250 & $17.3 \pm 0.2^{\mathrm{bc}}$ & $17.1 \pm 0.1^{\mathrm{bcd}}$ & $4.03 \pm 0.08^{\mathrm{abcd}}$ & $4.03 \pm 0.08^{\mathrm{bcd}}$ & $0.60 \pm 0.06^{\mathrm{c}}$ & $0.59 \pm 0.06^{\mathrm{c}}$ & $1.32 \pm 0.15^{\mathrm{bcd}}$ & $1.27 \pm 0.06^{\mathrm{bcd}}$ & $25.2 \pm 0.5^{\mathrm{abcd}}$ & $25.2 \pm 0.51^{\mathrm{bcd}}$ & $40.7 \pm 0.3^{\mathrm{c}}$ & $40.5 \pm 0.3^{c}$ \\
\hline & 500 & $14.3 \pm 0.8^{\mathrm{fg}}$ & $14.3 \pm 1.4^{\mathrm{f}}$ & $3.26 \pm 0.08^{\mathrm{fg}}$ & $3.24 \pm 0.08^{\mathrm{hi}}$ & $0.43 \pm 0.002^{\mathrm{gh}}$ & $0.43 \pm 0.01^{\mathrm{g}}$ & $0.97 \pm 0.10^{\mathrm{hi}}$ & $0.89 \pm 0.20^{\mathrm{ij}}$ & $20.4 \pm 0.5^{\mathrm{fg}}$ & $20.3 \pm 0.51^{\mathrm{hi}}$ & $31.5 \pm 0.7^{j}$ & $30.9 \pm 0.8^{\mathrm{i}}$ \\
\hline
\end{tabular}

Values are given as mean \pm SD of three replicates. Means in columns with different letters are significantly different at $\mathrm{P}<0.05$ by Duncan's Multiple Range Test. 
The data also indicated that cowpea leaflets have a well developed layer of water storage tissue, consisting of a layer 1-3 cells thick under the main vascular bundle in the midrib region. Foliar application of chitosan (especially at $250 \mathrm{mg} \mathrm{l}^{-1}$ ) increased the thickness of the leaf blade by increasing the thickness of the mesophyll tissue and (through the midrib region) the midrib vascular bundle. It also increased the thickness of the water storage tissue, and increased the area of xylem and phloem by stimulating pro-cambial activity in the midrib bundle during differentiation. Concerning the interaction between water stress and chitosan, the impact of chitosan appeared to be greatest at moderate water stress levels.

Although water deficit affects all stages of growth and development, grain yield is much more depressed than vegetative growth. Pod yield per plant and seed quality significantly decreased with increasing water deficit (Table 5), but foliar application of chitosan, especially at $250 \mathrm{mg} \mathrm{l}^{-1}$, reversed this and gave increases in yield and improved seed quality. The interactions treatment indicated that application of $250 \mathrm{mg} / \mathrm{l} \mathrm{CHI}$ under moderate and severe water deficit significantly increased the yield and its quality. Meanwhile the low and high concentration from $\mathrm{CHI}$ under moderate and severe water stress counteract the harmful effect of water deficit in this respect.

\section{Discussion}

The inhibiting effects of water stress on plant growth have previously been reported for soybean (Abdalla 2011), bambara groundnuts (Vurayai et al. 2011) and white lupins (Hefny 2011). It is well known that water stress conditions cause a multitude of molecular, biochemical and physiological changes, thereby affecting plant growth and development (Boutraa 2010). A decline in plant growth in response to water stress might be due either to decreases in cell elongation resulting from the inhibiting effect of water shortage on growthpromoting hormones which, in turn, lead to decreases in cell turgor, volume and eventually growth (Banon et al. 2006), and/or to the blocking up of xylem and phloem vessels, hindering translocation. Water-stress conditions cause a marked suppression in plant photosynthetic efficiency, mainly due to the closing of stomata, which limits $\mathrm{CO}_{2}$ diffusion into the leaf, the inhibition of ribulose-1,5-bisphosphate carboxylase/oxygenase (Rubisco), a non-stomatal factor (Lawlor \& Cornic 2002), and the impairment of ATP synthesis (Tezara et al. 1999). The depressive effect of water stress on growth parameters may also be attributed to a drop in leaf relative water content which reduces leaf turgor (unpublished data) and the assimilation of water and nitrogen compounds (Reddy et al. 2003), affecting the rate of cell division and enlargement. A reduction in the vegetative growth of plants under drought, in particular shoot growth, reduced cyclin-dependent kinase activity, resulting in slower cell division as well as inhibition of growth, and relatively severely reduced plant tissues, cell size, number of cells per unit or intercellular spaces (El-Beltagy et al. 1984). Drought stress also reduced the uptake of essential elements and photosynthetic capacity (Kandil et al. 2001) as well as the excessive accumulation of intermediate compounds such as reactive oxygen species (Yazdanpanah et al. 2011) which cause oxidative damage to DNA, lipid and proteins and consequently a decrease in plant growth. Finally, water stress leads to increases in abscisic acid levels in roots, which is transported from roots to shoot where it acts in the apical region of the plant as an antagonist of the auxine and cytokinin, responsible for growth and cell division, respectively (Abdalla 2011) as well as inhibiting DNA synthesis.

Foliar spraying of chitosan in most cases resulted in a significant increase in cowpea growth parameters under normal or stressed conditions, more pronounced at the intermediate concentration of $250 \mathrm{mg} \mathrm{l}^{-1}$. This result is similar in rice, where Chibu \& Shibayama (1999) showed that both 0.1 and $0.5 \%$ chitosan increased the dry weights of land rice c.v. Misatohatamochi, sweet pepper (Ghoname et al. 2010) and cucumber and radish plants 
(Farouk et al. 2008, 2011). The stimulating effect of chitosan on plant growth may be attributed to an increase in the availability and uptake of water and essential nutrients through adjusting cell osmotic pressure, and reducing the accumulation of harmful free radicals by increasing antioxidants and enzyme activities (Guan et al. 2009). Khan et al. (2002) added that foliar application of chitosan increased the net photosynthetic rates of soybean and maize, correlated with increases in stomatal conductance and transpiration rate, without any effects on intercellular $\mathrm{CO}_{2}$ concentration. This pattern of increases in net photosynthetic rate and stomatal conductance in the absence of any increase in intercellular $\mathrm{CO}_{2}$ concentration indicates that the increase in net photosynthetic rate is due to enhanced uptake of $\mathrm{CO}_{2}$ within the leaf that results in improved stomatal conductance, rather than due to having more open stomata. If an increase in stomatal aperture had been the primary cause, an increase in the leaf intercellular $\mathrm{CO}_{2}$ concentration would have been expected (Morison 1998). In addition, the positive effect of chitosan on plant growth may be due to its effect on increasing phosphorous content, as found in our investigation. Phosphorous is an essential nutrient playing an important role in the biosynthesis and translocation of carbohydrates, and necessary for stimulating cell division and forming DNA and RNA (Nijjar 1985).

Regarding the concentration of chlorophylls and carbohydrates, the present investigation showed that water stress decreased chlorophylls and total carbohydrates concentration. The decrease in chlorophyll content under drought is thus a commonly observed phenomenon (Nikolaeva et al. 2010, Kumar et al. 2011). The decrease in chlorophyll under water stress might be due to reduced synthesis of the main chlorophyll pigment complexes encoded by the $c a b$ gene family (Allakhverdiev et al. 2003), or to destruction of chiral macroaggregates of the light-harvesting chlorophyll ' $a$ ' or ' $b$ ' pigment protein complexes which protect the photosynthetic apparatus, or to oxidative damage of chloroplast lipids, pigments and proteins (Lai et al. 2007). An additional possibility is impairment in the supply of magnesium and iron to the leaves. Similarly, the reduction in total sugar content induced by water stress treatments may be due to its inhibitory effect on photosynthetic activities, photosynthetic pigment concentrations (Table 2) or the activity of ribulose diphosphate carboxylase leading to decreases in all sugar fractions (Stibrova et al. 1986). The effects of chitosan, especially at $250 \mathrm{mg} \mathrm{l}^{-1}$, in increasing chlorophylls and total carbohydrate contents were confirmed in cucumber (Farouk et al. 2008) and radish (Farouk et al. 2011). Chitosan may alleviate the water stress effect on photosynthetic pigments by enhancing endogenous levels of cytokinins, which stimulate chlorophyll synthesis. Chibu \& Shiayama (2001) referred these positive effects to the greater availability of amino compounds released from chitosan. Our results indicate significant increases in both nitrogen and potassium content in plant shoots (Table 3), which may be play an important role in increasing the number of chloroplasts per cell, cell size and number per unit area, as well as increased synthesis of chlorophyll (Possingham 1980).

Water stress affects the availability of nutrients in the soil by its effects on the solubility and precipitation of salt, and alters physiological processes within the plant, including nutrient uptake and translocation (Power 1990). Nutrient uptake by plants is generally decreased under water stress conditions owing to a substantial decrease in transpiration rates coupled with impaired active transport and membrane permeability (Levitt 1980) resulting in reduced rootabsorbing power. Nitrogen is a constituent of many plant cell components such as amino and nucleic acids, and hence its deficiency rapidly inhibits plant growth. The decrease in $\mathrm{N}$ content due to water stress has been reported in various crops including wheat (Singh \& Usha 2003) and in soybean (Tanguilig et al. 1987). Phosphorus is one of the most important nutrients in the growth and development of plants. It plays a key role in cellular energy transfer, respiration, photosynthesis. Phosphorus uptake decreases with decreasing soil moisture in various crops such as pepper (Turner 1985) and wheat (Ashraf et al. 1998). The role of chitosan in increasing ionic content may be due to its effects on stabilizing cellular membranes through increasing 
antioxidants substances, saving cell membranes from oxidative stress and hence improving plant cell permeability (unpublished data). This observation is supported by the results of Guan et al. (2009), where the application of chitosan significantly decreased lipid peroxidation by stimulating antioxidant enzymes, leading to decreased membrane permeability and improved function. Other reports have confirmed these results (Farouk et al. 2008, 2011; Ghoname et al. 2010).

The reduction in cowpea yield due to water stress has been reported before (Costa et al. 2008, Vurayai et al. 2011, Hefny 2011). In legume plants such as cowpea, seed yield is determined by three components: the number of pods per plant that reach maturity, the average number of seeds in each pod and dry weight of seeds. There are many hypotheses about the influence of water stress on yield. One of them suggests that water stress decreases the number of branches and leaves per plant (Table 1) as well as leaf area (shown in unpublished data), resulting in a reduction in the supply of carbon assimilate and photosynthetic rate as well as reduced radiation interception by plants and consequently less biomass produced as well as decreased translocation of photoassimulate towards the developing fruits (Kumar et al. 1994). Another possibility that also would reduce the yield due to water stress is by increasing the rate of flower and pod abortion (Liu et al. 2003). A decreased rate of carbohydrate flux from leaves to reproductive structures has been reported to control pod set in well-watered plants (Kokubun et al. 2001, Setter et al. 2001). Recent evidence supports this hypothesis. In maize, low water potential disrupted carbohydrate metabolism in ovaries by reducing the activity of acid invertase, the key enzyme catalyzing the breakdown of incoming sucrose during ovary and early seed development (Anderson et al. 2002). Song et al. (1998) showed that water stress induced swollen pollen and filament development, decreased filament fertility and resulted in reductions in grain number and weight per ear.

The increase in cowpea yield due to chitosan application may be due to its effects in stimulating physiological processes, improving vegetative growth, followed by active translocation of photoassimilates from source to sink tissues, increasing leaf-blade thickness as well as the dimensions of the vascular bundles, as indicated in our results. The increases in plant biomass may be due to improving photosynthetic machinery (Khan et al. 2002). Ghoname et al. (2010) also observed that foliar application of chitosan on sweet pepper increased significantly the number of fruits per plant and the mean weight of fruit, as well as quality characteristics such as total acidity, total soluble solid and ascorbic acid content in the fruit. The role of chitosan in alleviating the harmful effect of water stress on yield may be due to an increase in stomatal conductance and net photosynthetic $\mathrm{CO}_{2}$-fixation activity under water stress (Khan et al. 2002), and to its role in reducing transpiration to save water. Chitosan-treated stressed plants had a thicker entire-leaf lamina, upper epidermis and palisade mesophyll than untreated stressed plants. A thicker upper epidermis (including upper cuticle) and a thicker palisade parenchyma in these treatments may enhance survival and growth under water stress conditions by improving water relations and providing higher protection for the inner tissues (Bacelar et al. 2006), increasing yield.

It can be concluded that cowpea plants treated with chitosan induced the ability to grow under water stress conditions, perhaps because they can produce various metabolites which cause the closure of stomata, resulting in a reduction in transpiration.

\section{Acknowledgements}

We would to thanks all staff member of Agric. Botany Department, Faculty of Agriculture, Mansoura University, Egypt, in particular, Prof M.N. Helali for his helpful insights and critical reviews of the manuscript. 


\section{References}

Abdalla MM (2011) Beneficial effects of diatomite on the growth, the biochemical contents and polymorphic DNA in Lupinus albus plants grown under water stress. Agriculture \& Biology Journal of North America 2: $207-220$

Abdel-Mawgoud AMR, Tantawy AS, El-Nemr MA \& Sassine YN (2010) Growth and yield responses of strawberry plants to chitosan application. European Journal of Scientific Research 39 (1):161-168

Allakhverdiev I, Hayashi H, Nishiyama Y, Ivanov AG, Aliev JA, Klimov VV, Murata N \& Carpentier R (2003) Glycinebetaine protects the D1/D2/Cytb559 complex of photosystem II against photo-induced and heatinduced inactivation. Journal of Plant Physiology 160:41-49

Anderson MN, Asch F, Wu Y, Jnsen CR, Naested H, Mogensen VO \& Koch KE (2002) Soluble invertase expressionis an early target of drought stress during the critical, abortion-sensetive phase of young ovary development in maize. Plant Physiology 130: 591-604.

Ashraf MY, Ali SA, Bhatti AS (1998). Nutritional imblances in wheat (Triticum aestivum L.) genotypes grown at soil water stress. Acta Physiologia Plantarum 20 (3):307-310

Banon SJ, Ochoa J, Franco JA, Alarcon JJ \& Sanchez-Blanco MJ (2006) Hardening of coleander seedlings by deficit irrigation and low air humidity. Environmental \& Experimental Botany 56: 36-43

Bacelar EA, Santos DL, Moutinho-Pereira JM, Goncalves BC, Ferreira HF \& Correia CM (2006) Immediate response and adaptative strategies of three olive cultivars under constrasting water availability regimes: changes on structure and chemical composition of foliage and oxidative damage. Plant Science 170:596605

Bittelli M, Flury M, Campbell GS \& Nichols EJ (2001) Reduction of transpiration through foliar application of chitosan. Agricultural \& Forest Meteorology 107(3): 167-175

Boutraa T (2010) Improvement of water use efficiency in irrigated agriculture: a review. Journal of Agronomy 9: $1-8$

Carvalho IS, Ricardo CP\& Chaves M (2004) Quality and distribution of assimilates within the whole plant of lupines (Lupinus albus and L. mutabilis) influenced by water stress. Journal of Agronomy \& Crop Science 190: $205-210$

Chibu H \& Shibayama H (2001). Effects of chitosan applications on the growth of several crops. In: Uragami T, Kurita K, Fukamizo T(eds) Chitin and chitosan in life science. Yamaguchi, pp 235-239.

Chibu H \& Shibayama H (1999) Effects of chitosan applications on the early growth of several crops. Report of Kyushu Branch of the Crop Science Society of Japan 65: 83-87.

Cooper TG (1977) The tools of biochemistry. Wiley-Interscience. John Wiley \& Sons, New York.

Costa RCL, Lobato AKS, Oliveira Neto CF, Maia PSP, Alves GAR \& Laughinghouse HD (2008) Biochemical and physiological responses in two Vigna unguiculata (L.) Walp. cultivars under water stress. Journal of Agronomy 7: 98-101

Doares SH, Syrovets T, Wieler EW\& Ryan A (1995) Oligogalacturonides and chitosan activate plant defensive gene through the octadecanoid pathway. Proceedings of the National Academy of Sciences USA 92:40954098

EL-Beltagy AS, EL-Said MT, Sawsan MH, Hasniya MG \& Abo EL-Maksoud A (1984) Effect of different water regimes on tomato. Annals of Agricultural Science, Ain Shams University 29:1937-1956

Farouk S, Mosa AA, Taha AA, Ibrahim Heba M \& EL-Gahmery AM (2011) Protective effect of humic acid and chitosan on radish (Raphanus sativus L. var. sativus) plants subjected to cadmium stress. Journal of Stress Physiology \& Biochemistry 7(2):99-116

Farouk S, Ghoneem KM \& Ali Abeer A (2008) Induction and expression of systematic resistance to downy mildew disease in cucumber plant by elicitors. Egyptian Journal of Phytopathology 1-2: 95-111

Gaballah MS \& Moursy M (2004) Reflectants application for increasing wheat plant tolerance against salt stress. Pakistan Journal of Biolpgical Science 7:956-962

Ghoname AA, EL-Nemr MA, Abdel-Mawgoud AMR \& El-Tohamy WA (2010) Enhancement of sweet pepper crop growth and production by application of biological, organic and nutritional solutions. Research Journal of Agriculture \& Biological Science 6(7): 349-355.

Guan Ya-jing, Jin Hu, Xian-ju Wang \&Chen-xia Shao (2009) Seed priming with chitosan improves maize germination and seedling growth in relation to physiological changes under low temperature stress. Journal of Zhejiang University Science B 10(6):427-433

Hefny MM (2011) Agronomical and biochemical responses of white lupin Lupinus albus L. genotypes to contrasting water regimes and inoculation treatments. Journal of American Science 7(3):187-198

Jansen G (2008) Effects of temperature on yield and protein content of Lupinus angustifolius cultivars. In: Lupins for health and wealth. Proceedings of the $12^{\text {th }}$ International lupin conference. 14-18 September 2008. Fremantle, Western Australia.

Kalra YP (1998) Handbook of reference methods for plant analysis. CRC Press, Boca Raton, Florida, USA. 
Kandil SA, Abo-El-Kheir MSA \& El-Zeiny HA (2001) Response of some wheat cultivars to water stress imposed at certain growth stages. Egyptian Journal of Applied Science 16: 82-98

Yazdanpanah S, Baghizadeh A \& Abbassi F (2011). The interaction between drought stress and salicylic and ascorbic acids on some biochemical characteristics of Satureja hortensis. African Journal of Agricultural Research 6(4): 798-807

Khan MH, Singha KLB \& Panda SK (2002) Changes in antioxidant levels in Oryza sativa L. roots subjected to $\mathrm{NaCl}$ salinity stress. Acta Physiologia Plantarum 24:145-148.

Kokubun M, Shimada S \& Takahashi M (2001) Flower abortion caused by preanthesis water deficit is not attributed to impairment of pollen in soybean. Crop Science 41:1517-1521.

Kumar A, Singh DP \& Singh P (1994) Influence of water stress on photosynthesis, transpiration, water use efficiency and yield of Brassica juncea L. Field Crops Research 37:95-101

Kumar Ravi Ranjan, Krishna Karajol \& Naik GR (2011) Effect of polyethylene-glycol-induced water stress on physiological and biochemical responses in Pigeonpea (Cajanus cajan L. Millsp.). Recent Research in Science \& Technology 3(1):148-152

Lai Q, Zhi-yi B, Zhu-Jun Z, Qiong-Qiu Q \& Bi-Zeng M (2007) Effects of osmotic stress on antioxidant enzymes activities in leaf discs of PSAG12-IPT modified gerbera. Journal of Zheijang University of Science 8(7):458-464 doi:10.1631/jzus.2007.B0458.

Lawlor DW\& Cornic G (2002) Photosynthetic carbon assimilation and associated metabolism in relation to water deficits in higher plants. Plant Cell \& Environment 25: 275-294

Leung J\& Giraudat J (1998) Abscisic acid and signal transduction. Annual Review of Plant Physiology \& Plant Molecular Biology 49: 199-222.

Levitt J (1980) Responses of plants to environmental stresses. $2^{\text {nd }}$ ed., New York, Acad. Press.

Lichtenthaler HK\& Wellbum AR (1983) Determination of total carotenoids and chlorophylls A and B of leaves in different solvents. Biochemical Society Transactions 11:591-592

Liu F, Andersen MN \& Jensen CR (2003) Loss of pod set caused by drought stress is associated with water status and ABA content of reproductive structures in soybean. Functional Plant Biology 30:271-280

Lobato AKS, Costa RCL \& Oliveira Neto CF (2006) NR activity and RWC in Feijao-Caupi under water stress. In Proceedings of the 1st Congress Nacional de Feijao-Caupi and 6th Reuniao Nacional de Feijao-Caupi,22 26 May, Teresina, Brasil Empresa Brasileira de Agropecuaria, Teresina.

Lobato AKS, Oliveira Neto CF, Costa RCL, Santos Filho BG, Cruz FJR \& Laughinghouse HD (2008) Biochemical and physiological behaviour of Vigna unguiculata (L) Walp, under water stress during the vegetative phase. Asian Journal of Plant Science 7(1): 44-49.

Morison J IL (1998) Stomatal response to increased CO2 concentration. Journal of Experimental Botany 49:443452

Mullet JE \& Whitsitt MS (1996) Plant cellular responses to water deficit. Plant Growth Regulation 20(2):119-124

New N, Chandrkrachang S, \& Stevens WF (2004) Application of chitosan in Myanmar's agriculture sector, in: Proceedings of the Sixth Asia Pacific Chitin and Chitosan Symposium, May 23-26, The National Universityof Singapore, Singapore.

Nijjar GS (1985) Nutrition of fruit trees. Usha Raji Kumar, Kalyani, New Delhi, India

Nikolaeva MK, Maevskaya SN, Shugaev AG \& Bukhov NG (2010) Effect of drought on chlorophyll content and antioxidant enzyme activities in leaves of three wheat cultivars varying in productivity. Russian Journal of Plant Physiology 57: 87-95

Ogbonnaya CI, Sarr B, Brou C, Diouf O, Diop NN \& Roy-Macauly H (2003) Selection of cowpea in hydroponics, pots and field for drought tolerance. Crop Science 43:1114-1120

Possingham JV (1980) Plastid replication and development in the life cycle of higher plants. Annual Review of Plant Physiology 31: 113-129.

Power JF (1990). Role of moisture stress in plant nutritional function in crops as enhancers of nutrient use. Acad. Press, New York

Reddy TY, Reddy VR \& Anbumozhi N (2003) Physiological responses of groundnut (Arachis hypogaea L.) to drought stress and its amelioration: critical review. Plant Growth Regulation 41(3): 75-78

Sadasivam S \& Manickam A (1996) Biochemical methods. 2nd edition, New Age International. India.

Setter TL, Flannigan BA \& Melkonian J (2001) Loss of kernel set due to water deficit and shade in maize: carbohydrate supplies, abscisic acid, and cytokinins. Crop Science 41: 1530-1540

Shao H, Li-Ye C, Abdul Jaleel C, Manivannan P, Panneerselvam P \& Muig-An S (2009) Understanding water deficit stress-induced changes in the basic metabolism of high plants- biotechnologically and sustainably improving agriculture and the ecoenvironment in arid regions of the globe. Critical Reviews in Biotechnology 29(2): 131-151

Sheikha SA \& Al-Malki FM (2011) Growth and chlorophyll responses of bean plants to chitosan applications. European Journal of Scientific Research 50(1): 124-134

Singh B \& Usha K (2003) Salicylic acid induced physiological and biochemical changes in wheat seedlings under water stress. Plant Growth Regulation 39: 137-141. 
Somerville C \& Briscoe J (2001) Genetic engineering and water. Science 292: 2217.

Song FB, Ying DJ, Lie Z, Kun HG \& YiQing G (1998) Effect of water stress on maize pollen vigour and filament fertility. Acta Agronomica Sinica 24:368-373

Stibrova M, Doubravova M, Brezlova A \& Fridrich A (1986) Effects of heavy metals ions on growth and biochemical characteristics of photosynthesis of barley. Photosynthetica 20:416-425

Tambussi EA \& Bort $\mathrm{J}$ (2007) Water use efficiency in $\mathrm{C}_{3}$ cereals under Mediterranean conditions: a review of physiological aspects. Annals of Applied Biology 150:307-321

Tanguilig VC, Yambao EB, Tooles JCO \& Dedatta SK (1987) Water stress effects on leaf elongation, leaf water potential, transpirationand nutrient uptake of rice, maize and soybean. Plant \& Soil 103:155-168.

Tezara W, Mitchell VJ, Driscoll SD \& Lawlor DW (1999) Water stress inhibits plant photosynthesis by decreasing coupling factor and ATP. Nature 401: 914-917

Turner LB (1985) Changes in the phosphorous content of Capsicum annuam L) leaves during water stress. Journal of Plant Physiology 121: 429.

Vurayai R, Emongor V \& Moseki B (2011) Effect of water stress imposed at different growth and development stages on morphological traits and yield of bambara groundnuts (Vigna subterranean L. Verde). American Journal of Plant Physiology 6(1): 17-27.

\section{الملخص العربيى}

$$
\text { تحسين نمو وإنتاجية نبات اللوبيا النامي تحت ظروف الإجهاد المائي بو اسطة الكيتوزان }
$$$$
1 \text { قسم النبات الزراعي، كلية الزر اعة، جامعة المنصورة، } 2 \text { قسم النبات، المركز القومي للبحوث،الدقي }
$$

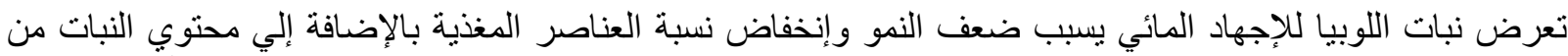

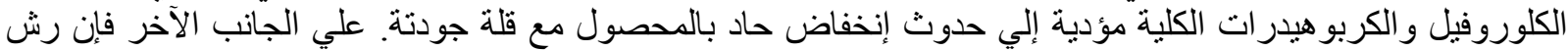

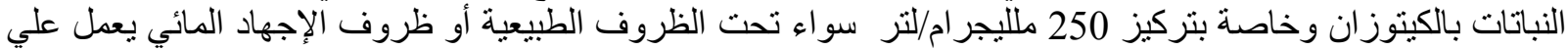

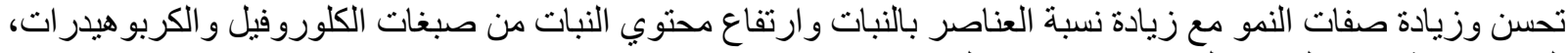

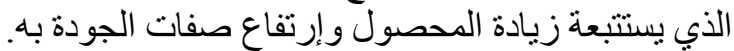

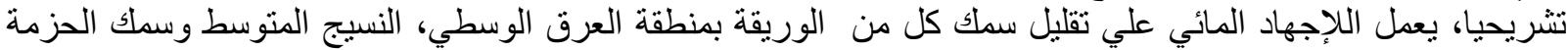

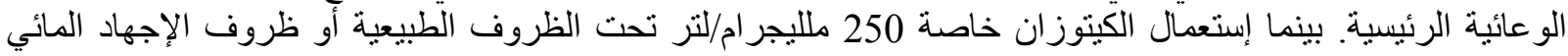

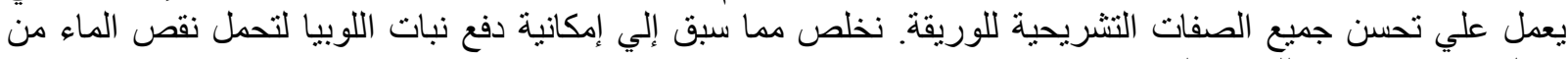
خلال إستخدام 250 ملليجر ام/لتر كيتوزان. 